\title{
On Mean Field Theories of Singlet Superconductivity in Extended Hubbard Models
}

\author{
J. Stolze \\ Institut für Physik, Universität Dortmund, Federal Republic of Germany \\ Received June 20, 1986
}

Hubbard models extended by attractive interactions between nearest neighbours have recently been discussed as models for heavy fermion superconductivity. Here, a comparison is carried through between mean field calculations at finite band width and exact results on ground state properties in the atomic limit (i.e. at zero band width). It turns out that the mean field approximation does not provide an appropriate description of the ground state of the system. This holds true for arbitrary band filling. For half-filled band, an extended mean field approximation is studied, allowing for both antiferromagnetism and (singlet) superconductivity. It is found that these two types of order do not coexist within mean field theory.

\section{Introduction}

Heavy fermion superconductivity has attracted considerable interest since its discovery some years ago. The large body of literature on the subject is reviewed in several recent articles or collections [1-3]. At the present time there seems to be no general consensus on the theoretical description of superconducting heavy fermion systems, and thus several model hamiltonians of varying complexity have been discussed. One of the simplest conceivable models is an extended Hubbard model, describing electrons moving in a (narrow) band and interacting by an on-site repulsion and a near-neighbour attraction. The attractive interaction may be introduced as a purely phenomenological parameter whose physical origin has to be clarified by considering a more detailed microscopic model. In two recent publications $[4,5]$, mean field approximations were developed for models of this type. In both cases an attractive interaction between electrons at nearest neighbour sites was introduced. In [5] the interaction was supposed to act only between electrons of opposite spin directions, whereas in [4] a spin-independent density-density interaction was assumed. The discovery of gapless or nearly gapless superconducting states was reported and nuclear spin relaxation experiments were interpreted. The aim of this paper is to compare these mean field results for narrow band extended Hubbard systems to recent exact results [6] on zero band width extended Hubbard systems.

To this end the ground state energy will be studied. For reasons of symmetry and convexity the ground state energy of a finite band width system (on a suitable lattice) should always be lower than that of the corresponding zero band width system. This property offers a simple way of judging the quality of mean field approximations. As mean field theories may be derived from variational principles for the energy or the free energy, they should provide reasonable values for these quantities, even if they fail to describe all details of the state of the system. Conversely, a failure of mean field theory to determine the energy correctly would cast serious doubts on mean field results for other quantities.

In Sect. II, the mean field theory of (singlet) superconductivity for the models of $[4,5]$ is developed from the variational principle. Systems of arbitrary particle density between zero and two electrons per site are discussed. The resulting "gap equations" for the system with spin-independent interaction differ slightly from those derived in [4]. However, it is found that the superconducting states found in mean field theory always have a higher ground state energy than the zero band width ground state. 
The zero band width ground state of the system with attractive interaction between opposite spins shows antiferromagnetic order. Thus one may think of improving the mean field theory of this model by additionally allowing for antiferromagnetic order. This is done in Sect. III, where only the case of one electron per lattice site will be treated for simplicity. It turns out that within the framework of mean field theory there is no coexistence of antiferromagnetism and superconductivity in this model.

In view of the negative results obtained in Sects. II and III it is hard to imagine how a (singlet) superconducting ground state might arise in an extended Hubbard model. Thus this model is probably not suitable for the description of heavy fermion superconductivity.

\section{Mean Field Theory of Superconductivity}

The Hubbard model is defined by the Hamiltonian

$H_{H}=-t \sum_{\sigma=\uparrow, \downarrow\langle i, j\rangle}\left(c_{i \sigma}^{+} c_{j \sigma}+c_{j \sigma}^{+} c_{i \sigma}\right)+U \sum_{i} n_{i \uparrow} n_{i \downarrow}$.

For definiteness, we consider a simple cubic lattice of $N$ sites; however, our treatment may be generalized to other "loose-packed" or " $A B$ " lattices (i.e. lattices consisting of two interpenetrating sublattices with each point of one sublattice surrounded by points of the other sublattice as nearest neighbours). On each lattice site $i$ there act two pairs of fermion operators $c_{i \sigma}^{+}, c_{i \sigma}(\sigma=\uparrow, \downarrow)$, furthermore

$n_{i \sigma}:=c_{i \sigma}^{+} c_{i \sigma}$.

$\sum_{\langle i, j\rangle}$ denotes summation over all nearest-neighbour pairs, where each pair is counted once. The first term in $H_{H}$ describes two degenerate tight binding bands with dispersion relation

$\varepsilon_{\mathbf{k}}^{0}=-2 t\left(\cos k_{x}+\cos k_{y}+\cos k_{z}\right)=:-t f(\mathbf{k})$.

(We set the volume of the elementary cube to unity.) $U$ describes the intra-atomic Coulomb repulsion $(U>0)$.

In order to obtain electron pairing, an attractive interaction must be added to (II.1). In [5] an interaction of the form

$H_{1}=-\frac{J}{2} \sum_{\langle i, j\rangle}\left(n_{i \uparrow} n_{j \downarrow}+n_{i \downarrow} n_{j \uparrow}\right) \quad(J>0)$

was introduced, describing an attraction between two electrons of opposite spin directions at neighbouring sites; a spin-independent interaction was used in [4]:

$H_{1}=-\frac{J}{2} \sum_{\sigma, \sigma^{\prime}} \sum_{\langle i, j\rangle} n_{i \sigma} n_{j \sigma^{\prime}}$.

(The model described by $H=H_{H}+H_{1}$ with $H_{1}$ given by (II.4) is usually called "extended Hubbard model".)

Mean field theories of interacting systems are most conveniently formulated using variational principles [7]. As we shall only discuss ground state properties, we may employ the Rayleigh-Ritz type inequality

$E(n N) \leqq\langle H\rangle_{0}$.

Here $E(n N)$ is the ground state energy of the extended Hubbard model Hamiltonian $H$ with $n$ electrons per lattice site. \langle\rangle$_{0}$ denotes the expectation value in the "ground state" of $H_{0}-\mu \hat{N}$, where $H_{0}$ is a simple effective Hamiltonian containing variational parameters and $\mu$ is the chemical potential which has to be adjusted according to the condition

$\langle\widehat{N}\rangle_{0}=n N$

on the expectation value of the total particle number operator $\hat{N}$. Variation with respect to a parameter $\lambda$ under the condition (II.6) then yields the stationarity condition

$$
0=\frac{\partial\langle H\rangle_{0}}{\partial \lambda} \frac{\partial\langle\hat{N}\rangle_{0}}{\partial \mu}-\frac{\partial\langle H\rangle_{0}}{\partial \mu} \frac{\partial\langle\hat{N}\rangle_{0}}{\partial \lambda}
$$

(for non-zero $\frac{\partial\langle\hat{N}\rangle_{0}}{\partial \mu}$; if $\langle\hat{N}\rangle_{0}$ does not depend on $\mu$, the condition (II.6) remains fulfilled anyway if $\lambda$ is varied).

In order to describe superconducting pairing in the Hamiltonian $H$, the effective Hamiltonian $H_{0}$ should allow for the existence of nonvanishing anomalous expectation values $\left\langle c_{i \uparrow}^{+} c_{i \downarrow}^{+}\right\rangle_{0}$ and $\left\langle c_{i \uparrow}^{+} c_{j \downarrow}^{+}\right\rangle_{0}$. We therefore introduce the following effective Hamiltonian:

$$
\begin{aligned}
H_{0}-\mu \hat{N}= & -t \sum_{\sigma} \sum_{\langle i, j\rangle}\left(c_{i \sigma}^{+} c_{j \sigma}+c_{j \sigma}^{+} c_{i \sigma}\right)-\mu \sum_{i \sigma} n_{i \sigma} \\
& +\sum_{i}\left(\Delta_{0} c_{i \downarrow} c_{i \uparrow}+\text { h.c. }\right) \\
& +\frac{1}{2} \sum_{\langle i, j\rangle}\left[\Delta_{1}\left(c_{i \downarrow} c_{j \uparrow}+c_{j \downarrow} c_{i \uparrow}\right)+\text { h.c. }\right] .
\end{aligned}
$$

$\Delta_{0}$ and $\Delta_{1}$ are variational parameters. It is more convenient to write (II.8) in $\mathbf{k}$-representation, using

$c_{i \sigma}^{+}=N^{-1 / 2} \sum_{\mathbf{k}} \mathrm{e}^{-i \mathbf{k} \mathbf{R}_{i}} c_{\mathbf{k} \sigma}^{+}$.

One then obtains

$H_{0}-\mu \hat{N}=\sum_{\mathbf{k}}\left\{\varepsilon_{\mathbf{k}} \sum_{\sigma} c_{\mathbf{k} \sigma}^{+} c_{\mathbf{k} \sigma}+g_{\mathbf{k}} c_{k \downarrow} c_{-k \uparrow}+g_{\mathbf{k}}^{*} c_{-k \uparrow}^{+} c_{k \downarrow}^{+}\right\}$, 
where

$\varepsilon_{\mathbf{k}}:=-t f(\mathbf{k})-\mu$,

$g_{\mathbf{k}}:=\Delta_{0}+\frac{\Delta_{1}}{2} f(\mathbf{k})$,

and $f(\mathbf{k})$ is defined by (II.2). The diagonalization of $H_{0}-\mu \hat{N}$ is performed by means of the standard Bogolyubov transformation to new fermion operators $b_{\mathbf{k} \sigma}, b_{\mathbf{k} \sigma}^{+}$

$c_{\mathbf{k} \sigma}=u_{\mathbf{k}} b_{\mathbf{k} \sigma}+\operatorname{sign}(\sigma) v_{\mathbf{k}} b_{-\mathbf{k}-\sigma}^{+}$,

with

$\operatorname{sign}(\sigma):= \begin{cases}+1 & \text { for } \sigma=\uparrow \\ -1 & \text { for } \sigma=\downarrow\end{cases}$

The $b$ operators fulfill fermion commutation relations if the parameters $u_{\mathbf{k}}$ and $v_{\mathbf{k}}$ fulfill the conditions

$u_{\mathbf{k}}=u_{-\mathbf{k}}, \quad v_{\mathbf{k}}=v_{-\mathbf{k}}$

and

$\left|u_{\mathbf{k}}\right|^{2}+\left|v_{\mathbf{k}}\right|^{2}=1$.

If $u_{\mathbf{k}}$ and $v_{\mathbf{k}}$ are chosen according to

$u_{\mathbf{k}}=2^{-1 / 2}\left[1+\frac{\varepsilon_{\mathbf{k}}}{\sqrt{\varepsilon_{\mathbf{k}}^{2}+\left|g_{\mathbf{k}}\right|^{2}}}\right]^{1 / 2}$

$v_{\mathbf{k}}=-2^{-1 / 2} \frac{g_{\mathrm{k}}^{*}}{\left|g_{\mathbf{k}}\right|}\left[1-\frac{\varepsilon_{\mathbf{k}}}{\sqrt{\varepsilon_{\mathbf{k}}^{2}+\left|g_{\mathbf{k}}\right|^{2}}}\right]^{1 / 2}$,

$H_{0}-\mu \hat{N}$ assumes diagonal form:

$H_{0}-\mu \hat{N}=\sum_{\mathbf{k}, \sigma}\left[E_{\mathbf{k}} b_{\mathbf{k} \sigma}^{+} b_{\mathbf{k} \sigma}+\varepsilon_{\mathbf{k}}\left|v_{\mathbf{k}}\right|^{2}+\operatorname{Re}\left(u_{\mathbf{k}} v_{\mathbf{k}} g_{\mathbf{k}}\right)\right]$,

with

$E_{\mathbf{k}}=+\left(\varepsilon_{\mathbf{k}}^{2}+\left|g_{\mathbf{k}}\right|^{2}\right)^{1 / 2}$.

As the excitation energies (II.19) are positive by construction, the ground state of $H_{0}-\mu \hat{N}$ simply is the $b$-vacuum.

Let us now sketch the zero temperature mean field theory of the Hamiltonian $H=H_{H}+H_{1}$, with $H_{1}$ given (II.3). Evaluating the expectation value $\langle H\rangle_{0}$ leads to the expression

$$
\begin{aligned}
\langle H\rangle_{0}= & 2 \sum_{\mathbf{k}} \varepsilon_{\mathbf{k}}^{0}\left|v_{\mathbf{k}}\right|^{2}+\frac{1}{N}\left(U-\frac{z J}{2}\right)\left(\sum_{\mathbf{k}}\left|v_{k}\right|^{2}\right)^{2} \\
& +\frac{U}{N}\left|\sum_{\mathbf{k}} v_{\mathbf{k}} u_{\mathbf{k}}\right|^{2}-\frac{J}{2 N} \sum_{\mathbf{k}, \mathbf{k}^{\prime}} v_{\mathbf{k}}^{*} u_{\mathbf{k}}^{*} v_{\mathbf{k}^{\prime}} u u_{\mathbf{k}^{\prime}} f\left(\mathbf{k}+\mathbf{k}^{\prime}\right) .
\end{aligned}
$$

$(z$ is the coordination number of the lattice, i.e. $z$ $=6$.)
The origin of the various terms of (II.20) may be easily determined by observing that

$\left\langle c_{\mathbf{k} \sigma}^{+} c_{\mathbf{k} \sigma}\right\rangle_{0}=\left|v_{k}\right|^{2}$

and

$\left\langle c_{\mathbf{k} \sigma}^{+} c_{-\mathbf{k}-\sigma}^{+}\right\rangle_{0}=\operatorname{sign}(\sigma) u_{\mathbf{k}}^{*} v_{\mathbf{k}}^{*}$.

Thus the first term of (II.20) obviously is the expectation value of the band term in $H_{H}$, and the second term arises from the "normal decoupling" of the interaction terms as products of particle density expectation values. The third and fourth terms contain anomalous expectation values; the fourth term may be simplified using the properties of the function $f(\mathbf{k})$ :

$\sum_{\mathbf{k}, \mathbf{k}^{\prime}} v_{\mathbf{k}}^{*} u_{\mathbf{k}}^{*} v_{\mathbf{k}^{\prime}} u_{\mathbf{k}^{\prime}} f\left(\mathbf{k}+\mathbf{k}^{\prime}\right)=z^{-1}\left|\sum_{\mathbf{k}} v_{\mathbf{k}} u_{\mathbf{k}} f(\mathbf{k})\right|^{2}$.

We now observe that the specific form (II.12) of $g_{\mathbf{k}}$ has not been used up to now. One may thus imagine a more general form of the effective Hamiltonian $H_{0}$, containing a completely arbitrary function $g_{\mathbf{k}}$. Variation with respect to this function then shows that $g_{\mathbf{k}}$ in fact has to assume the form (II.12) with $\Delta_{0}$ and $\Delta_{1}$ determined by

$\Delta_{0}=\frac{U}{N} \sum_{\mathbf{k}} v_{\mathbf{k}}^{*} u_{\mathbf{k}}^{*}=\frac{U}{N} \sum_{i}\left\langle c_{i \uparrow}^{+} c_{i \downarrow}^{+}\right\rangle_{0}$

and

$$
\begin{aligned}
\Delta_{1} & =-\frac{J}{N z} \sum_{\mathbf{k}} f(\mathbf{k}) v_{\mathbf{k}}^{*} u_{\mathbf{k}}^{*} \\
& =-\frac{J}{N z} \sum_{\langle i, j\rangle}\left(\left\langle c_{i \uparrow}^{+} c_{j \downarrow}^{+}\right\rangle_{0}+\left\langle c_{j 1}^{+} c_{i \downarrow}^{+}\right\rangle_{0}\right) .
\end{aligned}
$$

These two equations, together with the equation

$n=\frac{2}{N} \sum_{\mathbf{k}}\left|v_{\mathbf{k}}\right|^{2}$

for the particle density determine the values of $\Delta_{0}, \Delta_{1}$, and $\mu$. In order to discuss these equations it is convenient to rewrite them in integral form. To this end we introduce the dimensionless parameters

$$
\begin{array}{rlrl}
v: & =-\mu / 2 t & \\
\delta_{0}: & =\Delta_{0} / 2 t & \delta_{1}:=\Delta_{1} / 2 t \\
u: & =U / 4 t & j:=J / 12 t .
\end{array}
$$

The dimensionless density of states

$$
n(x)=\frac{1}{(2 \pi)^{3}} \int \mathrm{d}^{3} k \delta\left(x-\frac{1}{2} f(\mathbf{k})\right)=\frac{1}{2 \pi} \int_{-\infty}^{\infty} \mathrm{d} u \mathrm{e}^{\mathrm{i} u x} J_{0}^{3}(u)
$$


( $J_{0}$ is a Bessel function) is normalized according to

$\int_{-3}^{3} \mathrm{~d} x n(x)=1$.

Using furthermore the abbreviation

$E:=\left[(x+v)^{2}+\left|\delta_{0}+\delta_{1} x\right|^{2}\right]^{1 / 2}$,

the "gap equations" (II.24-26) may be written as

$$
\begin{aligned}
\delta_{0} & =-u \int_{-3}^{3} \mathrm{~d} x n(x)\left(\delta_{0}+\delta_{1} x\right) / E \\
\delta_{1} & =j \int_{-3}^{3} \mathrm{~d} x n(x) x\left(\delta_{0}+\delta_{1} x\right) / E \\
n & =1+\int_{-3}^{3} \mathrm{~d} x n(x)(x+v) / E
\end{aligned}
$$

In the same notation the mean field energy (II.20) may be rewritten as

$$
\begin{aligned}
\frac{\langle H\rangle_{0}}{N t}= & -2 \int_{-3}^{3} \mathrm{~d} x n(x) x(x+v) / E+(u-9 j) n^{2} \\
& +u\left|\int_{-3}^{3} \mathrm{~d} x n(x)\left(\delta_{0}+\delta_{1} x\right) / E\right|^{2} \\
& -j\left|\int_{-3}^{3} \mathrm{~d} x n(x) x\left(\delta_{0}+\delta_{1} x\right) / E\right|^{2}
\end{aligned}
$$

Obviously (II.30) and (II.31) always allow for the trivial solution $\delta_{0}=\delta_{1}=0$, with $v$ determined by (II.32). In this case the energy reduces to

$$
\frac{\langle H\rangle_{0}}{N t}=-2 \int_{-3}^{3} \mathrm{~d} x n(x) x \operatorname{sign}(x+v)+(u-9 j) n^{2}
$$

For $n=1$, (II.30) and (II.32) are solved by $\delta_{0}=v=0$, and (II.31) yields

$$
\left|\delta_{1}\right|=\left(j^{2} \gamma^{2}-1\right)^{1 / 2}
$$

(the phase of $\delta_{1}$ is obviously arbitrary), where

$$
\gamma:=\int_{-3}^{3} \mathrm{~d} x n(x)|x| \simeq 1.002
$$

(The numerical value was calculated using an approximation [8] to $n(x)$ in terms of elementary functions.) This solution, which was also found in [5], may be called gapless, as in this case the energy spectrum $E_{\mathbf{k}}$ starts at zero energy. The mean field energy is then given by

$$
\frac{\langle H\rangle_{0}}{N t}=-\frac{1}{j}+u-\left(9+\gamma^{2}\right) j \text {. }
$$

A solution resembling standard BCS superconductivity may also be found: for negative $u$ and $v=0$ one may solve the gap equations with $\delta_{1}=0, \delta_{0} \neq 0$. This "non-gapless" solution already occurs for infinitesimally negative $u$, whereas the gapless phase discussed above requires a finite $j$. We shall not further discuss the BCS-like solution, as we are only interested in the case of positive $u$.

For general values of $n, j$, and $u$, the equations (II.30-32) may be studied numerically; in this case both $\delta_{0}$ and $\delta_{1}$ will assume non-zero values. The mean field ground state energies obtained in this way may be compared to the ground state energy of the system in the exactly solvable limiting case of vanishing band width, which we shall now discuss.

The ground state energy of the system at $t=0$ is an upper bound to the (unknown) ground state energy at $t \neq 0$. This follows from two observations:

i) The ground state energy is a symmetric function of $t$ for an $A B$ lattice: a change of sign in $t$ may be cancelled by a change of sign of all fermion operators of one sublattice.

ii) The ground state energy is a concave function of $t$, as $t$ occurs in the Hamiltonian linearly [9].

This property of the ground state energy provides a valuable test for approximate theories of the $t \neq 0$ case: the ground state energy obtained by such an approximate treatment should at all events be lower than the atomic limit $(t=0)$ ground state energy. This applies in particular to theories like the mean field approximation, which are themselves based on energy minimum principles.

The possible ground states of rather general extended Hubbard model in the atomic limit were rigorously determined in [6]. Both models discussed in this paper (i.e. $H=H_{H}+H_{1}$ with $H_{1}$ given by (II.3) or (II.4)) belong to the class treated in [6] and thus the possible ground state configurations are known completely. Given the possible configurations it is easy to construct a ground state phase diagram showing which configuration is the ground state at a given point in parameter space.

The ground state phase diagram of the model given by (II.1) and (II.3) is depicted in Fig. 1 for $t=0$ and $U>0$. For positive $J$ the possible ground states are the completely empty and completely filled lattices, and a state with exactly one electron per lattice site and antiferromagnetic spin order. For values of $n$ different from 0,1 , or 2 , two of these phases coexist. The phases do not mix with each other but form spatially well-separated domains [6]. For negative $J$ (which we shall not further discuss) the antiferromagnetic order is replaced by ferromagnetic order, and the ferromagnetic phase is allowed to mix 


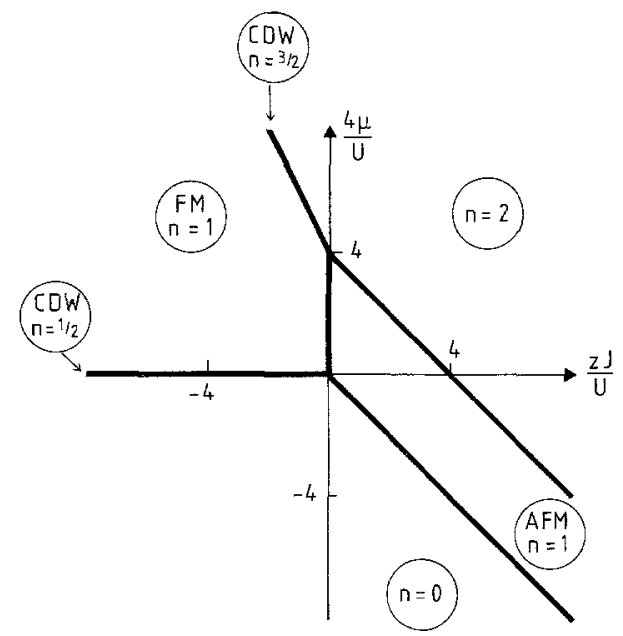

Fig. 1. Ground state phase diagram of the model defined by (II.1) and (II.3) in the atomic limit, for positive $U$. The abbreviations "CDW" and "(A)FM" mean "charge density wave" and "(anti-) ferromagnetism", respectively; $n$ denotes the average number of electrons per site and $z$ is the coordination number of the underlying $A B$ lattice

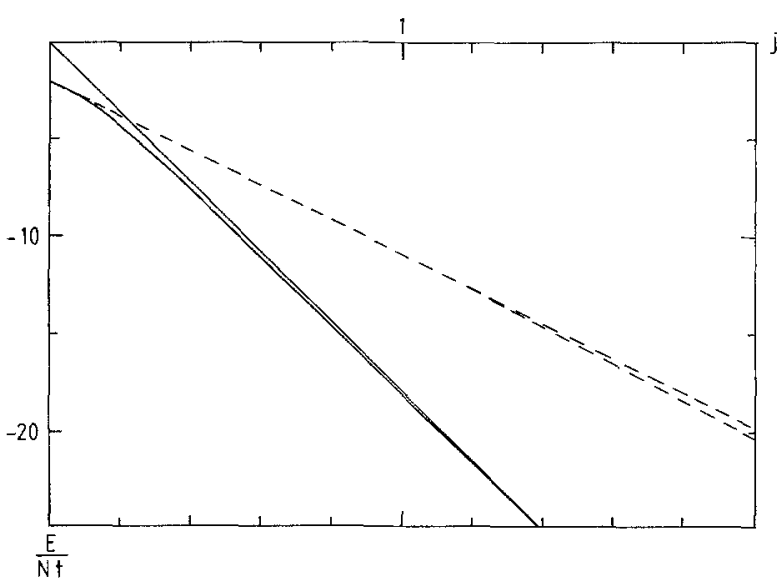

Fig. 2. Energy (per site, in units of $t$ ) for $n=1$ and $u=0$. Dashed lines represent the energy of the gapless solution, (II.37), (for $j>\gamma^{-1}$ ) and of the trivial solution (II.34) (upper curve). The upper solid line is the atomic limit ground state energy, the lower solid line is the energy of the antiferromagnetic solution from Sect. III

with the $n=0$ and $n=2$ phases due to the presence of additional "charge density wave" phases present at the boundaries in the phase diagram. These charge density wave phases consist of singly occupied and empty sites (or singly and doubly occupied sites) in an alternating fashion. Coexisting phases which are completely miscible may be easily affected by turning on the $t$ term in (II.1), whereas non-miscible phases should be more stable with respect to this perturbation [6]. Hence for sufficiently small band width (sufficiently heavy fermions) the atomic limit ground state should be a better approximation to the true ground state than the mean field ground state which allows only for superconducting order, but not for antiferromagnetic order. The atomic limit ground state energy thus should become lower than the mean field ground state energy for small enough $t$. (We note in passing that the atomic limit ground state energy is equal to the expectation value of the $t \neq 0$ Hamiltonian in the atomic limit ground state up to negligible interface contributions.)

The atomic limit ground state energy is easily determined (by collecting the contributions of all sites and bonds) once the ground state configurations are known. For a general $A B$ lattice with coordination number $z$ one obtains for the ground state energy per lattice site (with positive $U$ )

$\frac{E_{0}(t=0)}{N}= \begin{cases}(n-1)\left(U-\frac{z J}{2}\right) \theta(n-1) & \text { for } J \leqq 0 \\ -\frac{z J}{4} n+U(n-1) \theta(n-1, & \text { for } J \geqq 0 .\end{cases}$

( $\theta$ denotes the unit step function.)

Comparing (II.38) for $n=1$ and $J>0$ to the ground state energy of the gapless mean field solution and taking into account the restriction $j>\gamma^{-1}$ implied by (II.35) we see that the mean field ground state energy is never lower than the atomic limit ground state energy. Thus the mean field approximation fails to describe properly the situation at $n=1$ (see Fig. 2 as an example).

For $n \neq 1$ numerical solutions of (II.30-32) for various values of $u$ and $j$ did not yield a mean field ground state energy lower than the atomic limit ground state energy, thus indicating an inadequacy of the mean field approximation for all particle densities.

The reason for this failure might be sought in the neglect of magnetic order in the mean field approximation. The most easily visible (and probably quantitatively most important) effect of this neglect is the appearance of a term $-9 j n^{2}$ in (II.34) as compared to a term $-18 j n$ in $E_{0}(t=0) / N t$ (II.38). For $n=0$ and $n=2$ these terms are equal (as they should), whereas for $n=1$ they differ most drastically.

Two possible ways out of this conflict between mean field approximation for $t \neq 0$ and exact calculation at $t=0$ may be imagined: one may either take the spin dependence of the interaction seriously and try to develop a mean field approximation taking into account both antiferromagnetism and superconductivity (which we shall do in the next section), or one may use a spin-independent attractive interaction like (II.4) in order to avoid a magnetically ordered ground state in the atomic limit (which we shall do now). 
The mean field theory of the Hamiltonian $H$ $=H_{H}+H_{1}$ with $H_{1}$ given by (II.4) was discussed in [4]; the mean field self-consistency equations, however, were not obtained by a variational procedure but by ad hoc identification of the gap parameters with certain anomalous expectation values. It will turn out below that the variational procedure leads to self-consistency equations slightly different from the ones presented in [4].

The only difference between (II.3) and (II.4) is an attractive interaction term between electrons of equal spins at nearest neighbour sites. Evaluating the expectation value of this interaction in the ground state of the effective Hamiltonian (II.8), we obtain the two terms

$-\frac{J}{2 N}\left(z\left|\sum_{\mathbf{k}} v_{\mathbf{k}}^{2}\right|^{2}-\frac{1}{z}\left|\sum_{\mathbf{k}} f(\mathbf{k}) v_{\mathbf{k}}^{2}\right|\right)$.

The first of these terms is equal to one already present in (II.20); it is a product of density expectation values. The second term arises from decoupling the expectation value of the interaction in the form

$\left\langle c_{i \sigma}^{+} c_{j \sigma}\right\rangle_{0}\left\langle c_{j \sigma}^{+} c_{i \sigma}\right\rangle_{0}$.

(Anomalous decoupling of the additional interaction term is not taken into account, as this would lead to triplet pairing which was explicitly excluded.) The presence of terms of the type (II.40) might suggest the introduction of an additional variational parameter in the effective Hamiltonian, which would obviously represent an effective band width. However, as long as we are only interested in ground state properties, we only need the ground state of $H_{0}$ $-\mu \hat{N}$ and thus the band width of $H_{0}$ as an independent parameter may be "scaled away". Working at finite temperature would introduce another energy scale; in this case an effective band width of the effective Hamiltonian should be employed to get a consistent approximation.

Assuming $g_{\mathbf{k}}$ to be of the form (II.12) and varying $\Delta_{0}$ and $\Delta_{1}$ leads to two stationarity conditions which are consistently solved by

$\Delta_{1}=\frac{\frac{J}{N z} \sum_{\mathbf{k}} u_{\mathbf{k}} v_{\mathbf{k}} f(\mathbf{k})}{\frac{J}{2 t N z} \sum_{\mathbf{k}} v_{\mathbf{k}}^{2} f(\mathbf{k})-1}$

and either of the two values

$$
A_{0}=\frac{\frac{U}{N} \sum_{\mathbf{k}} u_{\mathbf{k}} v_{\mathbf{k}}}{1-\frac{J}{2 t N z} \sum_{\mathbf{k}} v_{\mathbf{k}}^{2} f(\mathbf{k})}
$$

or

$\Delta_{0}=\mu \Delta_{1} / 2 t$.

(Note that $\Delta_{0}$ and $\Delta_{1}$ are now supposed to be real.)

The condition (II.26) on the particle density of course has to be obeyed in addition. The denominator, by which $(I I .41,42)$ differ from the gap equations of [4] (and from (II.24, 25)) may be interpreted as a band width renormalization of the type discussed above. The solution described by (II.43) leads to a proportionality between $g_{\mathrm{k}}$ and $\varepsilon_{\mathrm{k}}$ and thus to a gapless excitation spectrum (II.19). In dimensionless form, equations (II.41, 42) read

$$
\begin{array}{r}
\delta_{1}=j \int_{-3}^{3} \mathrm{~d} x n(x) x\left(\delta_{0}+\delta_{1} x\right) / E \\
\left(1-j \int_{-3}^{3} \mathrm{~d} x n(x) x(x+v) / E\right) \\
\delta_{0}=-u \int_{-3}^{3} \mathrm{~d} x n(x)\left(\delta_{0}+\delta_{1} x\right) / E \\
\left(1-j \int_{-3}^{3} \mathrm{~d} x n(x) x(x+v) / E\right) .
\end{array}
$$

The mean field energy now reads

$$
\begin{aligned}
\frac{\langle H\rangle_{0}}{N t}= & -2 \int_{-3}^{3} \mathrm{~d} x n(x) x(x+v) / E+(u-18 j) n^{2} \\
& +u\left[\int_{-3}^{3} \mathrm{~d} x n(x)\left(\delta_{0}+\delta_{1} x\right) / E\right]^{2} \\
& -j\left[\int_{-3}^{3} \mathrm{~d} x n(x) x\left(\delta_{0}+\delta_{1} x\right) / E\right]^{2} \\
& +j\left[\int_{-3}^{3} \mathrm{~d} x n(x) x(x+v) / E\right]^{2}
\end{aligned}
$$

As before there is the trivial solution $\delta_{0}=\delta_{1}=0$, leading to

$$
\begin{aligned}
\frac{\langle H\rangle_{0}}{N t}= & -2 \int_{-3}^{3} \mathrm{~d} x n(x) x \operatorname{sign}(x+v)+(u-18 j) n^{2} \\
& +j\left[\int_{-3}^{3} \mathrm{~d} x n(x) x \operatorname{sign}(x+v)\right]^{2} .
\end{aligned}
$$

The gapless solution defined by (II.43) leads to

$\left|\delta_{1}\right|=\left[4 j^{2} \tilde{\gamma}^{2}-1\right]^{1 / 2}$

with

$\tilde{\gamma}:=\int_{-3}^{3} \mathrm{~d} x n(x) x \operatorname{sign}(x+v)$ 
Using (II.48) and (II.32), one obtains for the energy

$$
\begin{aligned}
\frac{\langle H\rangle_{0}}{N t}= & -\frac{1}{2 j}+(u-18 j) n^{2} \\
& +u(n-1)^{2}\left(4 j^{2} \tilde{\gamma}^{2}-1\right)-j \tilde{\gamma}^{2} .
\end{aligned}
$$

For $n=1$ this gapless solution coincides with the one given by (II.44, 45); for arbitrary $n$, numerical methods may again be used. Let us now discuss the atomic limit for comparison. The ground state phase diagram is given in Fig. 5a of [6]. For $U$ and $J$ positive, the possible ground states are the $n=0$ and $n=2$ states, and a paramagnetic state with one electron at each site; these states do not mix with each other. The ground state energy per site is given by

$$
\frac{E_{0}(t=0)}{N}=\left\{\begin{array}{l}
\frac{n}{2}(U-z J) \quad \text { for } z J \geqq 2 U \\
-\frac{n}{4} z J+\left(U-\frac{z J}{2}\right)(n-1) \theta(n-1)
\end{array}\right.
$$

for $z J \leqq 2 U$.

Again it is possible analytically to compare this result to the mean field ground state energy for $n=1$ and again the mean field ground state energy is higher than (II.51) whenever a nontrivial solution to the mean field equations exists. As before, numerical evaluations for $n \neq 1$ and various values of the coupling constants show that the atomic limit ground state is a better approximation to the true ground state than the mean field ground state.

The reason for this failure of the mean field approximation is again revealed by a closer look at the energy values for $n=1$. In this case the mean field ground state energy (II.50) contains a term $(u$ $-18 j$ ) arising from the density-density decoupling of the interaction terms. The corresponding terms in (II.51) (divided by $t$ ) are $2(u-18 j)$ for $9 j>u$ and $-18 j$ for $9 j<u$. We thus see that the atomic limit ground state lowers its energy by breaking translational invariance: for the case $9 j>u$ of weak Coulomb repulsion, a phase separation into $n=0$ and $n$ $=2$ phases takes place, making full use of the net attractive interaction. For $9 j>u$ the atomic limit ground state avoids the strong Coulomb repulsion by having each site occupied by exactly one electron of definite spin. (The antiferromagnetic ground state discussed earlier of course may be viewed as another example of this phenomenon.)

The fact that the mean field theories developed in $[4,5]$ fail to approximate the ground state energy of course casts some doubts upon evaluations of more complicated physical quantities in the framework of these theories.

\section{Superconductivity and Antiferromagnetism}

In this section we are going to discuss the model Hamiltonian $H=H_{H}+H_{1}$ with $H_{1}$ given by (II.3) in a mean field approximation taking into account antiferromagnetism, as well as superconductivity as possible types of order. For simplicity we shall restrict ourselves to the case $n=1$, where the atomic limit yields pure antiferromagnetism and where the simple mean field theory of the preceding section yields gapless superconductivity. Accordingly we define the following effective Hamiltonian

$$
\begin{aligned}
H_{0}= & -t \sum_{\mathbf{k}, \sigma} f(\mathbf{k}) c_{\mathbf{k} \sigma}^{+} c_{\mathbf{k} \sigma}+\frac{\Delta_{1}}{2} \sum_{\mathbf{k}} f(\mathbf{k})\left(c_{\mathbf{k} \downarrow} c_{-\mathbf{k} \uparrow}+\text { h. c. }\right) \\
& -m \sum_{i} \mathrm{e}^{\mathrm{i} \mathbf{Q} \mathbf{R}_{i}}\left(n_{i \uparrow}-n_{i \downarrow}\right)
\end{aligned}
$$

with $\mathbf{Q}:=(\pi, \pi, \pi)$. The "staggered molecular field" term in (III.1) may be rewritten as

$\sum_{i} \mathrm{e}^{\mathrm{i} \mathbf{Q} \mathbf{R}_{i}}\left(n_{i \uparrow}-n_{i \downarrow}\right)=\sum_{\mathbf{k}}\left(c_{\mathbf{k} \uparrow}^{+} c_{\mathbf{k}-\mathbf{Q} \uparrow}-c_{\mathbf{k} \downarrow}^{+} c_{\mathbf{k}-\mathbf{Q} \downarrow}\right)$.

The diagonalization of this effective Hamiltonian is most conveniently performed in two steps. In a first step we eliminate the terms which do not conserve particle number by introduction of new fermion operators $b_{\mathbf{k} \sigma}, b_{\mathbf{k} \sigma}^{+}$. This leads to

$H_{0}=\sum_{\mathbf{k}, \sigma}\left(E_{\mathbf{k}} b_{\mathbf{k} \sigma}^{+} b_{\mathbf{k} \sigma}-m \operatorname{sign}(\sigma) b_{\mathbf{k} \sigma}^{+} b_{\mathbf{k}-\mathbf{Q} \sigma}\right)$

with

$E_{\mathbf{k}}:=-f(\mathbf{k}) \sqrt{t^{2}+\Delta_{1}^{2} / 4}$.

A second transformation to fermion operators $a_{\mathbf{k} \sigma}, a_{\mathbf{k} \sigma}^{+}$leads to

$H_{0}=\sum_{\mathbf{k} \sigma} \Lambda_{\mathbf{k}} a_{\mathbf{k} \sigma}^{+} a_{\mathbf{k} \sigma}$

where

$\Lambda_{\mathbf{k}}:=-\operatorname{sign}(f(\mathbf{k})) \sqrt{E_{\mathbf{k}}^{2}+m^{2}}$.

Equation (III.6) represents an effective quasiparticle band which is split up due to the antiferromagnetic superstructure imposed. The ground state of (III.5) has the lower subband filled and the upper subband empty. This state corresponds to $n=1$ by particlehole symmetry.

The complete transformation between the original $c$ operators and the new $a$ operators may be written as follows:

$$
\begin{aligned}
c_{\mathbf{k}, \sigma}= & A_{\mathbf{k}}(+,+) a_{\mathbf{k}, \sigma} \\
& -\operatorname{sign}(\sigma) \operatorname{sign}(f(\mathbf{k})) A_{\mathbf{k}}(+,-) a_{\mathbf{k}-\mathbf{Q}, \sigma} \\
& -\operatorname{sign}(\sigma) A_{\mathbf{k}}(-,+) a_{-\mathbf{k},-\sigma} \\
& +\operatorname{sign}(f(\mathbf{k})) A_{\mathbf{k}}(-,-) a_{-\mathbf{k}-\mathbf{Q},-\sigma}
\end{aligned}
$$


where

$$
\begin{aligned}
A_{\mathbf{k}}(\rho, \tau):= & \frac{1}{2}\left[1+\rho t /\left(t^{2}+\Delta_{1}^{2} / 4\right)^{1 / 2}\right]^{1 / 2} \\
& \cdot\left[1+\tau E_{\mathbf{k}} / \Lambda_{\mathbf{k}}\right]^{1 / 2}
\end{aligned}
$$

(and where " \pm 1 " has been abbreviated " $\pm "$ in (III.7)).

To evaluate $\langle H\rangle_{0}$ one may use the following expectation values

$$
\begin{aligned}
& \left\langle c_{\mathbf{k} \sigma}^{+} c_{\mathbf{k}^{\prime} \sigma^{\prime}}\right\rangle_{0}=\frac{1}{2} \delta_{\sigma \sigma^{\prime}}\left\{\delta_{\mathbf{k}, \mathbf{k}^{\prime}}\left(1+\frac{t f(\mathbf{k})}{\left|\Lambda_{\mathbf{k}}\right|}\right)\right. \\
& \left.\quad+m \operatorname{sign}(\sigma) \delta_{\mathbf{k}, \mathbf{k}^{\prime}-\mathbf{Q}} t /\left(t^{2}+\Delta_{1}^{2} / 4\right)^{1 / 2}\left|\Lambda_{\mathbf{k}}\right|\right\}, \\
& \left\langle\mathbf{c}_{\mathbf{k} \uparrow}^{+} \mathbf{c}_{\mathbf{k}^{\prime} \downarrow}^{+}\right\rangle_{0}=-\frac{\Delta_{1} \operatorname{sign} f(\mathbf{k})}{4\left(t^{2}+A_{1}^{2} / 4\right)^{1 / 2}} \delta_{\mathbf{k},-\mathbf{k}^{\prime}}
\end{aligned}
$$

to obtain

$$
\begin{aligned}
\frac{\langle H\rangle_{0}}{N}= & -t^{2} N^{-1} \sum_{\mathbf{k}} f^{2}(\mathbf{k}) /\left|A_{\mathbf{k}}\right|+\frac{1}{4}(U-z J / 2) \\
& -\frac{1}{4}(U+z J / 2) \frac{m^{2} t^{2}}{t^{2}+\Delta_{1}^{2} / 4}\left(N^{-1} \sum_{\mathbf{k}}\left|\Lambda_{\mathbf{k}}\right|^{-1}\right)^{2} \\
& -\frac{J}{8 z} \frac{\Delta_{1}^{2} / 4}{t^{2}+\Delta_{1}^{2} / 4}\left(N^{-1} \sum_{\mathbf{k}}|f(\mathbf{k})|\right)^{2}
\end{aligned}
$$

Employing the notation introduced in (II.27) and the abbreviations

$\bar{m}:=m / t$

and

$\Lambda(x):=\left[\left(1+\delta_{1}^{2}\right) 4 x^{2}+\bar{m}^{2}\right]^{1 / 2}$,

the following stationarity conditions (with respect to $\delta_{1}$ and $\bar{m}$ ) are obtained for $\langle H\rangle_{0}$ :

$1=(2 u+18 j) \int_{-3}^{3} \mathrm{~d} x n(x) / \Lambda(x)$

or

$\bar{m}=0$

and

$2 j \gamma^{2}=\int_{-3}^{3} \mathrm{~d} x n(x) \Lambda(x)$

or

$\delta_{1}=0$

or

$\delta_{1} \rightarrow \infty$.

The condition (III.14b) immediately leads to the old gapless solution (II.35) which was already discussed; (III.15c) is possible only if $t \rightarrow 0$, but for this case the

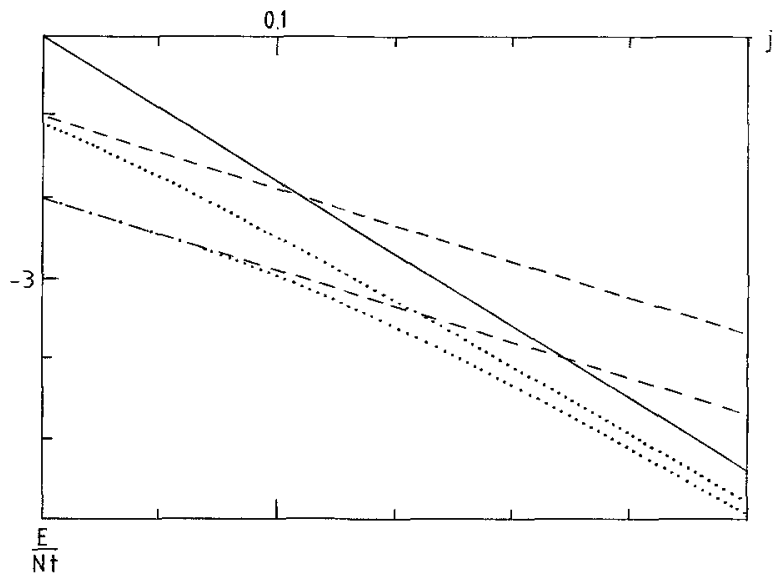

Fig. 3. Comparison of the atomic limit ground state energy (solid line), the trivial mean field solution (dashed lines) and the antiferromagnetic mean field solution from Sect. III (dotted lines) for $n$ $=1$ and $u=0$ or 1 . The higher mean field energies correspond to $u=1$. Note that the energy of the trivial mean field solution is rigidly shifted by $u$. (The same holds true for the energy of the gapless mean field solution for $j>\gamma^{-1}$.)

exact solution is known. A "purely antiferromagnetic" solution is implied by (III.15b). In this case (III.14b) has a solution for every positive value of ( $u$ $+9 j$ ). The energy of this state (per site, in units of $t$ ) is always lower than the atomic limit ground state energy; for $t \rightarrow 0$ the two energies become asymptotically equal, as they should. In Figs. 2 and 3 the energies of the gapless, antiferromagnetic, and trivial mean field solutions are compared to the atomic limit ground state energy.

A solution fulfilling (III.14a) and (III.15a) would describe coexistence of superconductivity and antiferromagnetism. Such a solution, however, does not exist. This may be shown by writing (III.15a) in the form

$2 j \gamma^{2}=\bar{\Lambda}$

where the bar denotes an averaging procedure of obvious definition. Employing Jensen's inequality [10] on averages of convex functions, one obtains from (III.14a)

$1=(2 u+18 j) \overline{\left(\Lambda^{-1}\right)} \geqq(2 u+18 j)(\bar{\Lambda})^{-1}=\frac{u}{j \gamma^{2}}+\frac{9}{\gamma^{2}}$

(III.17)

which is obviously impossible since $\gamma$ is close to unity.

To conclude, let us stress that the demonstrated failures of mean field theory of course do not prove the nonexistence of superconductivity in extended Hubbard models of the type discussed here. However, it is quite unclear how superconductive order 
might be described in such models, if not by some kind of mean field theory. Therefore other models should be (and are, of course, cf. e.g. the reviews [2, $3]$ ) considered for the theoretical description of the phenomena observed in heavy-fermion superconductors.

I am grateful to Gerd Czycholl for drawing my attention to the articles $[4,5]$ and for some helpful discussions.

This work has considerably benefited from numerous clarifying discussions with Uwe Brandt, to whom I wish to express my sincere thanks.

\section{References}

1. Stewart, G.R.: Rev. Mod. Phys. 56, 755 (1984)

2. Kasuya, T., Saso, T. (eds.): Theory of heavy fermions and valence fluctuations. In: Springer Series in Solid-State Sciences. Vol. 62. Berlin, Heidelberg, New York: Springer-Verlag 1985
3. Czycholl, G.: Phys. Rep. (to be published)

4. Miyake, K., Matsuura, T., Jichu, H.: Prog. Theor. Phys.72, 652 (1984)

5. Gulàcsi, M., Gulàcsi, Zs.: Solid State Commun. 56, 1059 (1985)

6. Brandt, U., Stolze, J.: Z. Physik B - Condensed Matter 62 , 433 (1986)

7. Cf. e.g. Girardeau, M.D., Mazo, R.M.: Advances in Chemical Physics. Vol. XXIV, p. 187 (1973) for a discussion of the BCS theory of superconductivity from this point of view

8. Jelitto, R.J.: J. Phys. Chem. Solids 30, 609 (1969)

9. For the free energy this may be derived from the PeierlsBogolyubov variational principle (see [7]), for the ground state energy see Ferrell, R.A.: Phys. Rev. Lett. 1, 443 (1958)

10. Hardy, G.H., Littlewood, J.E., Pólya, G.: Inequalities. Cambridge: Cambridge University Press 1967

\section{J. Stolze}

Institut für Physik

Universität Dortmund

Otto-Hahn-Strasse

D-4600 Dortmund 50

Federal Republic of Germany 